\title{
A 78-year-old woman with lethargy and a positive urine culture
}

\author{
Alon Vaisman MD, Wayne L. Gold MD, Jerome A. Leis MD
}

A 78-year-old woman residing in a long-term care facility is transferred to the emergency department because of generalized weakness and a urine culture that yielded a multidrugresistant strain of Escherichia coli. She recently experienced a self-limited febrile gastrointestinal illness during an enteric outbreak in her long-term care facility. The urine culture was performed 3 days before transfer to investigate her fever. She has no dysuria, urgency or increased frequency of urination, or suprapubic pain.

On examination, the patient is lethargic. Her blood pressure is $86 / 50 \mathrm{~mm} \mathrm{Hg}$, her heart rate is 110 beats/min, and her temperature is $36.7^{\circ} \mathrm{C}$. Her jugular venous pressure is below the sternal angle. There is no suprapubic or flank tenderness. She does not have an indwelling urinary catheter. A urine specimen obtained in the emergency department appears cloudy. You consider the significance of the positive urine culture and whether it represents a urinary tract infection or asymptomatic bacteriuria.

\section{Does this patient have a urinary tract infection or asymptomatic bacteriuria?}

The patient has asymptomatic bacteriuria, which is defined as a significant growth of bacteria ( $>10^{5}$ colony forming units $/ \mathrm{mL}$ of urine) in an appropriately collected specimen from a patient without signs or symptoms attributable to urinary tract infection..$^{1}$ The prevalence of asymptomatic bacteriuria increases with age and occurs in up to $50 \%$ of women and $35 \%$ of men residing in long-term care facilities. ${ }^{1,2}$

Prospective studies involving residents without indwelling urinary catheters in long-term care facilities have not established a relation between bacteriuria and nonurinary symptoms such as anorexia, changes in mental status, malaise, weakness or falls. ${ }^{2,3}$ Despite these findings, urine cultures are frequently ordered and antimicrobial therapy is prescribed because of false perceptions that changes in functional status are linked to the presence of bacteriuria. ${ }^{2}$

\section{If the patient had been unable to communicate her symptoms, how could the likelihood of urinary tract infection be assessed?}

In this situation, urinalysis may be useful in excluding urinary tract infection. A prospective study involving 101 residents of long-term care facilities with suspected urinary tract infection showed that a negative urine dipstick test for leukocyte esterase and nitrite had a negative predictive value for urinary tract infection of $100 \%$ (95\% confidence interval [CI] 74\%-100\%), essentially excluding urinary tract infection. ${ }^{4}$ Conversely, a positive urinalysis was unhelpful for diagnosing urinary tract infection (positive predictive value $45 \%, 95 \%$ CI $34 \%-56 \%)^{4}$ because pyuria is common among older patients. Similarly, the presence of malodorous or cloudy urine should not be relied on to diagnose urinary tract infection because these findings are nonspecific and may occur for many reasons, including intravascular volume depletion.

For patients with a positive urinalysis, physical examination should be performed to assess suprapubic pain, costovertebral tenderness or fever. However, because of age-related changes in temperature, elevations in temperature below those classically defined as fever or even hypothermia may be the only sign of infection among older patients. ${ }^{6}$ Even if fever is present, alternate sources must be excluded. Using standardized criteria for diagnosing urinary tract infection, ${ }^{1}$ a prospective study that included 372 febrile episodes among patients without catheters in a long-term care facility found that urinary infection contributed to less than $10 \%$ of the episodes of fever. ${ }^{7}$

Even after careful assessment, some uncertainty will exist for a subset of patients with bacteriuria who cannot communicate symptoms and who have experienced a change in status without an alternate explanation.
Competing interests: None declared.

This article has been peer reviewed.

Correspondence to: Jerome A. Leis, jerome.leis@utoronto.ca

CMAJ 2013. DOI:10.1503 /cmaj.121860 
Would this patient benefit from treatment for asymptomatic bacteriuria?

Five prospective randomized controlled trials have not shown any benefit in treating asymptomatic bacteriuria. ${ }^{1,8,9}$ Despite these findings, about half of patients with asymptomatic bacteriuria are prescribed antimicrobial therapy, which may result in unintended negative consequences. ${ }^{2,10}$ A retrospective chart review involving 172 residents with asymptomatic bacteriuria in long-term care facilities found an 8.5-fold increased risk of Clostridium difficile infection within 3 months of inappropriately receiving antimicrobial therapy. ${ }^{10}$ Prospective studies have also shown an increase in adverse drug effects and the isolation of increasingly resistant bacteria among patients who received antimicrobial therapy for asymptomatic bacteriuria compared with those who did not. ${ }^{8}$

In a recent randomized prospective trial involving 673 young women with asymptomatic bacteriuria, the occurrence of symptomatic urinary tract infection in the subsequent year was significantly higher among those who received treatment for asymptomatic bacteriuria than among those who did not $(48.6 \%$ v. $13 \%, p<$ 0.001). ${ }^{9}$ This study supports the theory that antimicrobial therapy for asymptomatic bacteriuria increases a patient's susceptibility to urinary tract infection by altering the normal intestinal and vaginal flora, which can lead to overgrowth of other uropathogens. ${ }^{9}$

\section{Should the long-term care facility's staff be advised to screen the patient's urine for bacteriuria in the future?}

Screening for asymptomatic bacteriuria is not indicated for older people living in the community or long-term care facilities, women with diabetes or people with spinal cord injury or an in situ catheter. ${ }^{1,8}$ Screening should only be conducted in situations where treatment is indicated.

Randomized controlled trials support screening for and treatment of asymptomatic bacteriuria in pregnant women, before transurethral resection of the prostate, and before other urologic procedures in which mucosal bleeding is anticipated. ${ }^{1}$

\section{Case revisited}

The patient's urinalysis was positive for leukocytes and nitrites. In the absence of localized urinary symptoms, her lethargy was attributed to intravascular volume depletion related to her recent enteric infection. The positive urine culture was considered to represent asymptomatic bacteriuria and was not repeated. Antimicrobial therapy was not prescribed. Saline was administered intravenously, and her functional status improved. She returned to the long-term care facility 72 hours later in stable condition.

\section{References}

1. Nicolle LE, Bradley S, Colgan R, et al. Infectious Diseases Society of America guidelines for the diagnosis and treatment of asymptomatic bacteriuria in adults. Clin Infect Dis 2005;40:643-54.

2. Walker S, McGeer A, Simor AE, et al. Why are antibiotics prescribed for asymptomatic bacteriuria in institutionalized elderly people? A qualitative study of physicians' and nurses' perceptions. CMAJ 2000;163:273-7.

3. Boscia JA, Kobasa WD, Abrutyn E, et al. Lack of association between bacteriuria and symptoms in the elderly. Am J Med 1986; 81:979-82.

4. Juthani-Mehta M, Tinetti M, Perrelli E, et al. Role of dipstick testing in the evaluation of urinary tract infection in nursing home residents. Infect Control Hosp Epidemiol 2007;28:889-91.

5. Midthun SJ, Paur R, Lindseth G. Urinary tract infections. Does the smell really tell? J Gerontol Nurs 2004;30:4-9.

6. Norman DC. Fever in the elderly. Clin Infect Dis 2000;31:148-51.

7. Orr PH, Nicolle LE, Duckworth H, et al. Febrile urinary tract infection in the institutionalized elderly. Am J Med 1996;100:71-7.

8. Nicolle LE, Mayhew WJ, Bryan L. Prospective randomized comparison of therapy and no therapy for asymptomatic bacteriuria in institutionalized elderly women. Am J Med 1987;83:27-33.

9. Cai T, Mazzoli S, Mondaini N, et al. The role of asymptomatic bacteriuria in young women with recurrent urinary tract infections: To treat or not to treat? Clin Infect Dis 2012;55:771-7.

10. Rotjanapan P, Dosa D, Thomas KS. Potentially inappropriate treatment of urinary tract infections in two Rhode Island nursing homes. Arch Intern Med 2011;171:438-43.

Affiliations: From the Department of Medicine (Vaisman, Gold, Leis), University of Toronto; and the Division of Infectious Diseases (Gold, Leis), University Health Network, Toronto, Ont.

Contributors: Jerome Leis and Wayne Gold contributed to the conception of the article, and all authors performed the literature review. Alon Vaisman drafted the manuscript, which all authors revised. All of the authors gave final approval of the version submitted for publication.

Decisions is a series that focuses on practical evidence-based approaches to common presentations in primary care. The articles address key decisions that a clinician may encounter during initial assessment. The information presented can usually be covered in a typical primary care appointment. Articles should be no longer than 650 words, may include one box, figure or table and should begin with a very brief description ( 75 words or less) of the clinical situation. The decisions addressed should be presented in the form of questions. A box providing helpful resources for the patient or physician is encouraged. 